\title{
Device-independent, real-time identification of bacterial pathogens with a metal oxide-based olfactory sensor
}

\author{
M. Bruins • A. Bos $\cdot$ P. L. C. Petit • K. Eadie $\cdot$ A. Rog $•$ \\ R. Bos • G. H. van Ramshorst • A. van Belkum
}

Received: 23 December 2008 / Accepted: 3 January 2009/Published online: 4 February 2009

(C) The Author(s) 2009. This article is published with open access at Springerlink.com

\begin{abstract}
A novel olfactory method for bacterial species identification using an electronic nose device called the MonoNose was developed. Differential speciation of microorganisms present in primary cultures of clinical samples could be performed by real-time identification of volatile organic compounds (VOCs) produced during microbial replication. Kinetic measurements show that the dynamic changes in headspace gas composition are orders of magnitude larger than the static differences at the end of fermentation. Eleven different, clinically relevant bacterial species were included in this study. For each of the species, two to eight different strains were used to take intra-species biodiversity into account. A total of 52 different strains were measured in an incubator at $37^{\circ} \mathrm{C}$. The results show that the diagnostic specificities varied from $100 \%$ for Clostridium difficile to $67 \%$ for Enterobacter cloacae with an overall average of $87 \%$. Pathogen identification with a MonoNose can be achieved within 6-8 h of inoculation of the culture broths. The diagnostic specificity can be improved by broth modification to improve the VOC production of the pathogens involved.
\end{abstract}

\footnotetext{
M. Bruins $\cdot$ A. Bos $(\triangle) \cdot$ R. Bos

C-It BV, Marspoortstraat 2, 7201 JB, Zutphen, The Netherlands

e-mail: Albert.Bos@C-it.nl
}

M. Bruins • P. L. C. Petit • K. Eadie • A. Rog • A. van Belkum

Erasmus MC, Department of Medical

Microbiology and Infectious Diseases,

University Medical Centre, 's Gravendijkwal 230,

3015 CE Rotterdam, The Netherlands

G. H. van Ramshorst

Erasmus MC, Department of Surgery,

University Medical Centre, 's Gravendijkwal 230,

3015 CE Rotterdam, The Netherlands

\section{Introduction}

Bacterial identification in the medical microbiology laboratory is still firmly based on old-fashioned biochemical reactions. From the ages of Pasteur and Koch onwards, medical microbiologists have relied on classical culture-based methods in order to confirm bacterial infections and identify the pathogens involved. Most infectious agents are still primarily detected using classical methods that involve liquid or solid semisynthetic growth media [1]. Serological methods for the detection of such pathogens are often lacking in sensitivity and specificity and data generated by the novel generation of expensive molecular tests need to be interpreted with equal caution [2]. Even so, given the clinical impact of bacterial infections in general, methods that reliably speed up the diagnostic process and limit costs are still eagerly awaited. Culture has the major advantage that living organisms are obtained for downstream characterisation, including antimicrobial susceptibility testing and epidemiological typing of the organism. This is an argument in favour of new methods that combine classical culture with procedures that enhance the speed of bacterial species identification. Currently, microbiology laboratories employ classical, growthbased, fermentative species-identification schemes that can be performed either manually or in an automated fashion using instruments such as the bioMérieux VITEK $($ or the Beckon-Dickinson Phoenix $(\mathcal{C}$. Molecular nucleic acid amplification tests and biophysical procedures, including mass spectrometry, could encompass novel, more real-time methods of bacterial species identification, although this would require additional handling of positive cultures [3]. In conclusion, real-time species identification during primary cultivation of 
clinical samples would be of added value for controlling costs and optimising patient care in clinical institutions. Odour-based assays could potentially fill this diagnostic niche. General interest in the classification of microorganisms on the basis of odour production has recently increased because of the introduction of so-called electronic nose devices.

Differential speciation of micro-organisms present in primary cultures of clinical samples could be performed by real-time identification of volatile organic compounds (VOCs) produced during microbial replication. Classical culture has been combined with such olfactory measurements in the past [4]. It needs to be emphasised that these experiments always involved cultivation endpoint measurements. However, by continuous sampling of a culture's headspace, the kinetics of the synthesis of VOCs can be monitored in real time. The complex bacteriological VOC signals can be defined by a gas measurement technique involving metal-oxide (MO) sensors as used in the present study.

We have developed a broadly applicable, inexpensive and highly responsive sensor system, called the MonoNose, which uses real-time VOC pattern recognition and the matching of the measured dynamic olfactory pattern with previously identified reference patterns. This system is analogous to the kinetics of human odour recognition [5]. The MonoNose technology facilitates the timely identification of bacterial species and, thereby, the clinical differentiation of medically relevant pathogens. In this article, we show that several bacterial species can be distinguished on the basis of MonoNose-mediated identification of growth stage-specific VOC production.

\section{Materials and methods}

\section{Electronic nose (MonoNose)}

A set of 30 custom-designed electronic nose devices were manufactured by $\mathrm{C}$-it (Zutphen, The Netherlands). These MonoNoses employ a single metal oxide-type semiconductor gas sensor. A scheme of a MonoNose and the 30 hand-manufactured devices in operation in an incubator are depicted in Fig. 1. The sensor system itself and the accompanying real-time pattern recognition algorithms are described in more detail elsewhere (manuscript submitted).

For each experiment a MonoNose device was fitted with a disposable sterile syringe needle and a sterile HEPA filter with $45-\mu \mathrm{m}$ pore size to prevent crosscontamination. Humidity influences the semiconductor response values [6]. All devices were operated in an incubator at a constant ambient temperature of $37^{\circ} \mathrm{C}$ in order to keep the relative humidity in the headspace constant during all experiments.

\section{Bacterial species}

Eleven different, clinically relevant bacterial species were included in this study. For each of the species two to eight individual, genotypically distinct strains were used to take intra-species biodiversity into account. The 52 strains used are listed in Table 1. The strains were obtained from both commercial sources (American Type Culture Collection; ATCC) and Erasmus MC reference collections. For all strains, the nature of the species was reconfirmed by
Fig. 1 a Schematic representation of a MonoNose device for measuring bacterial volatile organic compound (VOC) production in the broth' headspace over prolonged periods of time. The sensor is a commercially available metal oxide-based micro-device. b Experimental set-up with 30 MonoNose devices in operation in an incubator. The sensors are serially connected and all data are assembled on a simple portable computer. The vials in the photo are standard BDBACTEC $^{\text {TM_Plus-Anaerobic/F }}$ disposable bottles
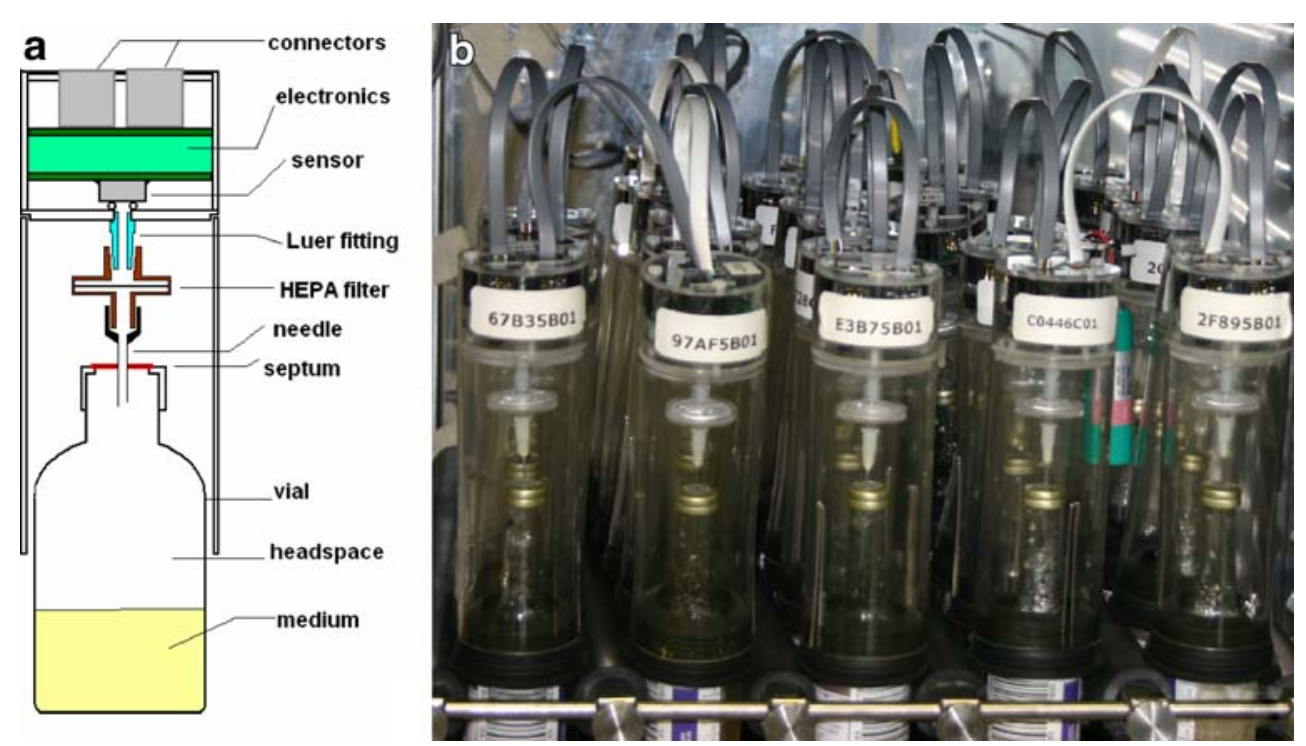
Table 1 Overview of the bacterial species and strains used

\begin{tabular}{ll}
\hline Species & Strains \\
\hline $\begin{array}{l}\text { Clostridium } \\
\text { difficile }\end{array}$ & AN59, AN63 \\
$\begin{array}{c}\text { Enterobacter } \\
\text { cloacae }\end{array}$ & ATCC13047, B33386, B33449 \\
Enterococcus & ATCC19433, ATCC29212, \\
faecalis & ATCC51299, ATCC7080, P794805, \\
& VanB, B33432, B33438 \\
Escherichia coli & 31972, 31995, ATCC25922, \\
& ATCC35218, B31938, ATCC35150(ETEC) \\
Klebsiella oxytoca & 32341, ATCC700324, B33516, B33510, \\
& F54, loes \\
Klebsiella & ATCC13882, ATCC13883, \\
pneumoniae & ATCC35657, ATCC700603, P79789 \\
Proteus mirabilis & ATCC25933, ATCC7002, B33183, \\
& B33505, B33546 \\
Pseudomonas & ATCC10145, ATCC27853, \\
aeruginosa & ATCC9027, P798326 \\
Salmonella & ATCC13076, mrcl \\
enteriditis & ATCC13311, ATCC14028, ATCC49416 \\
Salmonella & \\
typhimurium & 857S, 863S, 865S, 920S, ATCC25923, \\
Staphylococcus & ATCC29213, ATCC29737, ATCC33862 \\
aureus & \\
\hline
\end{tabular}

VITEK (C) analysis before inclusion in the present study. Culture broths $(50 \mathrm{ml})$ were inoculated with $100 \mu \mathrm{l}$ of a physiological salt solution with a bacterial density of \pm 1.5 McFarland and linked to the MonoNose.

Culture broths and chemicals

A broad range of commercially available culture broths were tested to identify those with maximum bacterial VOC production. These explorative tests were conducted with a single strain per species. The broths were also spiked with additional chemicals. These additions consisted of $1 \mathrm{ml}$ of a solution with a concentration of $0.1,1.0$ or $5.0 \mathrm{mM}$ of, for instance, metal salts (ammonium nickel [II] sulphate, chromium [III] potassium sulphate, cobalt [II] sulphate, copper [II] chloride, copper [II] sulphate, iron [III] chloride, lithium acetate, manganese [II] sulphate, molybdenum [IV] sulphide, silver nitrate and zinc sulphate), fatty acids (butanoic acid, caproic acid, lactic acid, propionic acid and valeric acid) and alcohols (butanol, ethanol, methanol and 2propanol). All separate chemicals were purchased from Sigma (Dordrecht, The Netherlands). As Escherichia coli and Klebsiella oxytoca strains produce VOCs that are efficiently detected by the MonoNose and, hence, produce "strong" signals, these two bacterial species were used to examine the influence of amino acid and $\mathrm{NaCl}$ concen- trations by varying the concentrations between 2.5 and 7.5 g. $1^{-1}$ and 1 and 9 g..$^{-1}$ respectively.

\section{Data processing}

During each experiment a set of 2,200-2,500 data points was collected (corresponding with an incubation of 12 $14 \mathrm{~h}$ ), starting within $15 \mathrm{~min}$ of the moment of inoculation of the medium. To test the device and time independency all strains were measured three times on different days and with different devices. The measured data were pre-processed and the resulting times-series data were analysed using a Sliding Window-Minimum Variance Matching adaptation of the Dynamic Time Warping algorithm $[7,8]$.

\section{Results}

Culture broths

The results of the exploratory tests with various broths revealed that the composition and dynamics of the headspace were co-determined by both the broth composition and the bacterial species in question. Although the different chemical additives caused changes in the VOCs produced, only one of the chemicals tested gave a significantly better result than was obtained in the standard broth. All other additions either generated no improvement at all or only improved the signal obtained for one species while deteriorating others, which invariably resulted in a worse overall result. These tests do, however, clearly reveal that the production of VOCs can be influenced by changes in the media, as the observed features change with the different additives. The BD-BACTECTM_Plus-Anaerobic/F Medium with the addition of $0.1 \mathrm{mM} \mathrm{FeCl}_{3}$ was selected to perform the high-throughput measurements, as this medium gave the best discriminating abilities of the broths tested.

Among the bacterial species tested, two response modes could be identified. Species either generated strong signals, probably because of the production of large amounts of detectable VOCs, or the signals remained low during the entire measurement process. The group of species provoking strong signals will be further referred to as the "strong" group, and suffered from a squashing effect because of the particulars of the measuring electronics: signal curves relatively quickly topped off due to saturation of the measurement signals.

Amino acid and salt concentrations showed a clear optimum, which differed for the species tested. The surface plots showing the results are depicted in Fig. 2. 
Bacterial species identification

Bacteria were cultured in the BD-BACTECTM-PlusAnaerobic/F Medium with the addition of $0.1 \mathrm{mM} \mathrm{FeCl}_{3}$ and the production of VOCs was measured continuously with the MonoNose. Figure 3 illustrates typical experimental results. Figure $3 \mathrm{a}$ is an example of a strong response in which the maximum amplitudes of the sensor reached the upper limits of the measuring range. Figure $3 b$ is an example of a weak response in which the maximum amplitudes of the sensor are found to remain near the
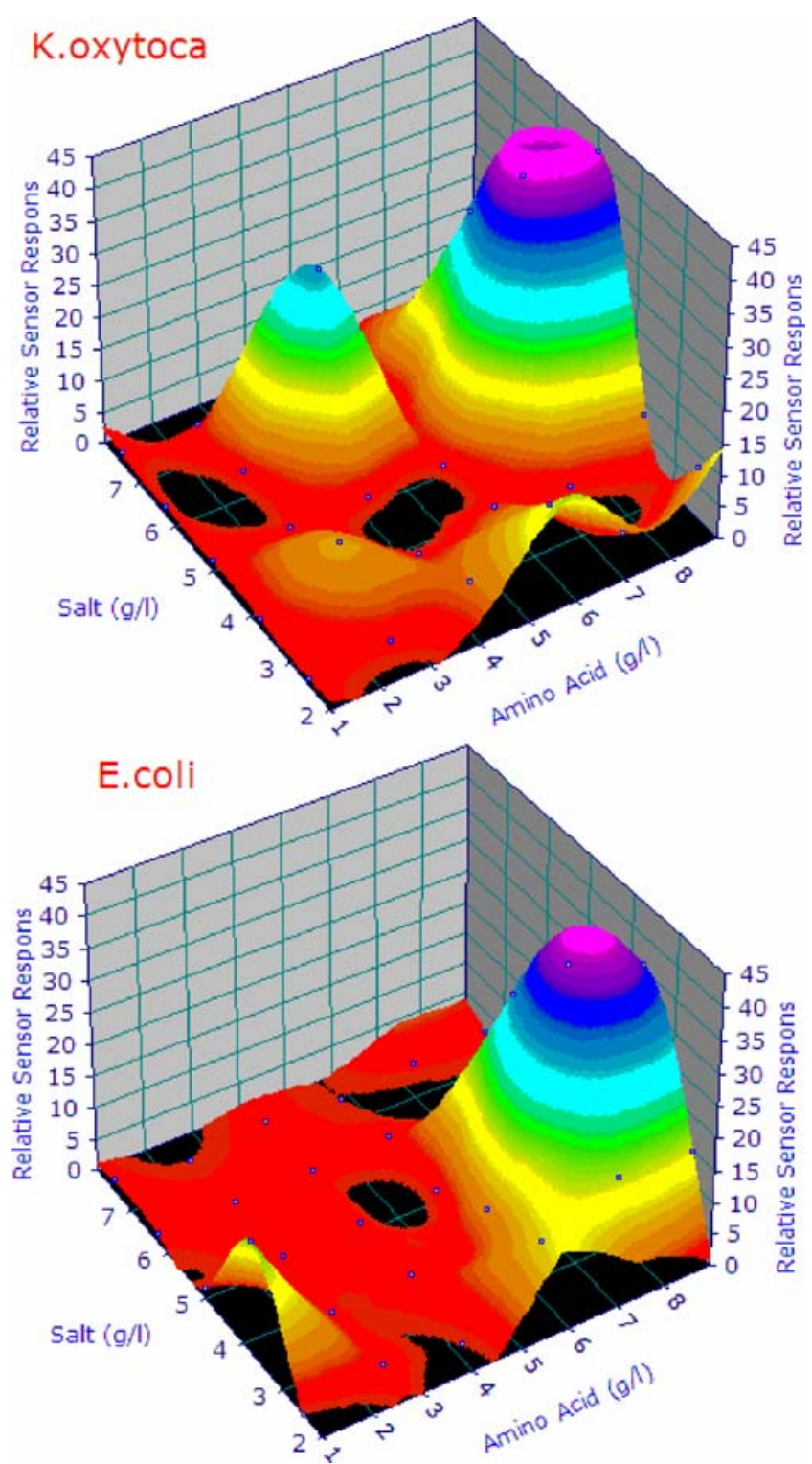

Fig. 2 The surface plot of the maximum sensor amplitude for $E$. coli and $K$. oxytoca, as a function of the combined amino acid and $\mathrm{NaCl}$ concentrations. Maxima and minima are clearly shown, as is the difference in optimal concentrations for these two species. Note that the major difference is reflected by a bi-modal response curve for $K$. oxytoca lower limit of the measuring range. It must be emphasised that bacterial identification can be adequately based upon both the strong and the weak signals with comparable specificity. The group of strong bacterial reactors was found to consist of the species Proteus mirabilis, Klebsiella spp., E. coli, Salmonella spp., E. cloacae and Clostridium difficile. The weak group was found to consist of Staphylococcus aureus, Enterococcus faecalis and Pseudomonas aeruginosa. For each species, a characteristic sub-feature was derived from a single experiment. Sub-features for the two Salmonella and the two Klebsiella species were very similar. Therefore, these species cannot yet be distinguished and had to be grouped at the genus level. Examples of these subfeatures are given in Fig. $3 c$ and d. The sub-features were subsequently "generalised" by matching and averaging within a species group. With the "generalised" sub-features, the classification process was iterated. The results in Table 2 show that the test specificity varies from $100 \%$ for C. difficile to $67 \%$ for E. cloacae with an overall average of $87 \%$. All characteristic sub-features were observed between 6 and $8 \mathrm{~h}$ after initial inoculation.

\section{Discussion and conclusions}

The majority of papers describing the classification of bacteria with electronic nose devices appeared around the turn of the century [9-12]. In most cases, very good diagnostic results were achieved and the bright prospects for the applications were hinted at. In all cases, however, the analysis models used a single sensing device. None of the papers addressed the crucial aspect of applicability and reproducibility when using a larger series of independent devices. This seriously hampers the broad applicability and hence the commercial success of such methodologies. Although the older approaches allowed for establishing proof of principle, results came at the great expense of painstakingly calibrating for each measuring device. In order to achieve similar results with other devices, the same amount of work needed to be performed for each unit. For practical application, it is necessary to ascertain that the reproducibility between a set of devices is sufficient to allow the development of analysing algorithms that do not depend on intensive, individual calibration of the measuring devices. If individual calibration is required to adapt an analysis model to a specific hardware device, the added effort prohibits a realistic application. The current data demonstrate that the MonoNose hardware and times-series analysis software give good results over a representative set of 30 independently produced hardware devices and 9 bacterial species. All measurements have been conducted with different MonoNoses and the results show that the features measured are interchangeable among the different 

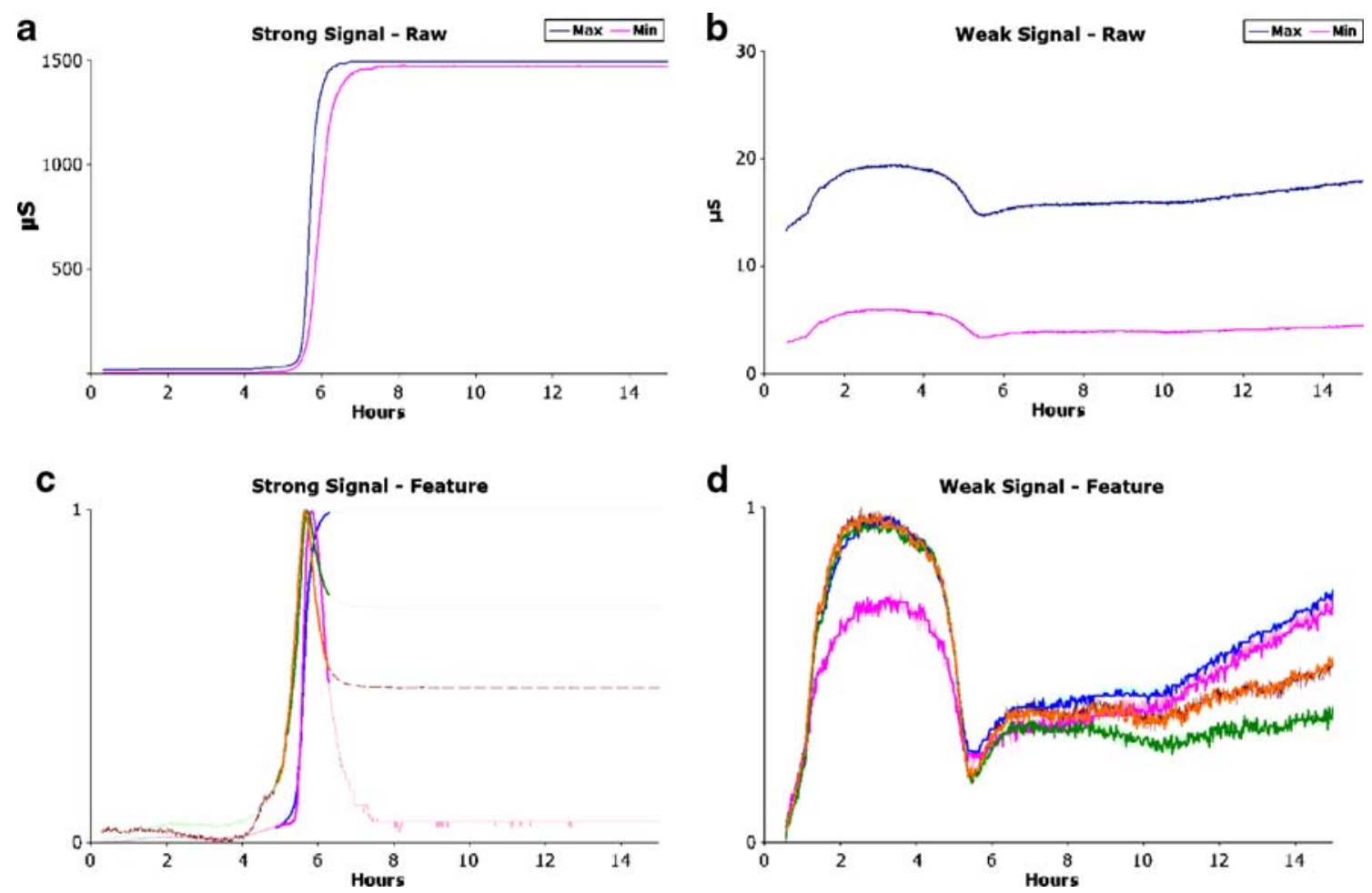

Fig. 3 a, b Experimental output of the VOC sensing during bacterial cultivation. a Typical strong result for $E$. coli. b Typical weak result for $S$. aureus. Note the difference in amplitudes between the strong and weak results. Species-specific signatures were derived from the VOC detection curves on the basis of Sliding Window Minimum Variance

MonoNoses, rendering obsolete the need for the individual calibration of each MonoNose.

This study shows that the MonoNose can identify pathogenic bacteria during the first few hours of growth on the basis of prior calibration data generated by other MonoNoses. Most identification systems in use today follow Bayes theorem and, depending on the number of tests used, obtain a score of $<90 \%$ (e.g. the bioMerieux API-50 test), higher only when significant numbers of additional tests are included. When using generalised

Matching and Dynamic Time Warping. c Selected E. coli feature projected on a normalised $E$. coli experiment. d Selected $S$. aureus feature projected on a normalised $S$. aureus experiment. Note the reproducible difference in time frames between the two features

features calculated on the basis of the VOC response curves an average identification rate of $87 \%$ was achieved. It is expected that this can be optimised further when the detection limits of the MonoNose are expanded. The availability of a more universal culture broth will further enhance the possibilities of the MonoNose to become a fast, reliable and inexpensive detection method for pathogenic bacteria. The study also demonstrates that the development of the metabolising behaviour of growing bacteria over time gives distinct and sometimes reversible features that

Table 2 MonoNose-based identification result for the bacterial species test panel

\begin{tabular}{|c|c|c|c|c|c|}
\hline & \multirow[t]{2}{*}{ Number of measurements } & \multirow[t]{2}{*}{ Unclassifiable } & \multicolumn{2}{|c|}{ Classified } & \multirow[t]{2}{*}{ Percentage correct } \\
\hline & & & Correct & Error & \\
\hline Clostridium difficile & 4 & 0 & 4 & 0 & 100 \\
\hline Enterobacter cloacae & 6 & 0 & 4 & 2 & 67 \\
\hline Enterococcus faecalis & 16 & 2 & 12 & 2 & 86 \\
\hline Escherichia coli & 12 & 1 & 9 & 2 & 82 \\
\hline Klebsiella spp & 22 & 0 & 21 & 1 & 95 \\
\hline Proteus mirabilis & 10 & 0 & 8 & 2 & 80 \\
\hline Pseudomonas aeruginosa & 8 & 3 & 5 & 0 & 100 \\
\hline Salmonella spp & 10 & 1 & 7 & 2 & 78 \\
\hline Staphylococcus aureus & 16 & 0 & 14 & 2 & 88 \\
\hline Overall & 104 & 7 & 84 & 13 & 87 \\
\hline
\end{tabular}


are traceable using a single-sensor electronic nose employing thermal cycling combined with software analysis.

With the broth used in the present experiments not all important pathogen species can be identified. Therefore, future work will focus on optimisation of the culture broth with a new MonoNose that has a larger dynamic measuring range. The amino acid and salt variations showed different optimal mixtures for the different organisms, meaning that changing the broths can greatly improve the result, but mostly the increase in one species will mean a decrease in another. Different clinical materials such as urine, blood and wound exudates also need to be tested to determine the influence of the sample matrix on the measurement. Ideally, the next generation of electronic noses will not only facilitate bacterial species identification after culture, but also the characterisation of bacterial cells present in non-processed clinical materials.

\section{Conflict of interest None.}

Open Access This article is distributed under the terms of the Creative Commons Attribution Noncommercial License which permits any noncommercial use, distribution, and reproduction in any medium, provided the original author(s) and source are credited.

\section{References}

1. Remans JJ, Verboom P, Stijnen T, Hakkaart-van Roijen L, Goessens W, Verbrugh HA et al (2008) Rapid identification and antimicrobial susceptibility testing reduce antibiotic use and accelerate pathogen-directed antibiotic use. J Antimicrob Chemother 61(2):428-435

2. Nowakowska D, Colón I, Remington JS, Grigg M, Golab E, Wilczynski J et al (2006) Genotyping of Toxoplasma gondii by multiplex PCR and peptide-based serological testing of samples from infants in Poland diagnosed with congenital toxoplasmosis. J Clin Microbiol 44(4):1382-1389. doi:10.1128/JCM.44.4.13821389.2006

3. Vanlaere E, Sergeant K, Dawyndt P et al (2008) Matrix-assisted laser desorption ionisation-time-of-flight mass spectrometry of intact cells allows rapid identification of Burkholderia cepacia complex. J Microbiol Methods 75(2):279-286

4. Casalinuovo IA, Pierro D, Bruno E, Francesco P, Coletta M (2006) Experimental use of a new surface acoustic wave sensor for the rapid identification of bacteria and yeasts. Lett Appl Microbiol 42(1):24-29. doi:10.1111/j.1472-765X.2005.01792.x

5. Haddad R, Khan R, Takahashi YK, Mori K, Harel D, Sobel N (2008) A metric for odorant comparison. Nat Methods 5:425-429. doi:10.1038/nmeth.1197

6. Pearce TC, Schiffman SS, Nagle HT, Gardner JW (2003) Handbook of machine olfaction. Wiley-VCH, Weinheim

7. Latecki LJ, Megalooikonomou V, Wang Q, Lakaemper R, Ratanamahatana CA, Keogh E (2005) Elastic partial matching of time series. Jorge A et al (eds) Knowledge discovery in databases: PKDD 2005. Lecture Notes in Computer Science. Springer, Berlin, pp 577-584

8. Berndt D, Clifford J (1994) Using dynamic time warping to find patterns in time series. AAAI-94 Workshop on Knowledge Discovery in Databases (KDD-94), Seattle, Washington

9. Guernin N, Ratcliffe M, Spencer-Phillips PTN, Howe RA (2001) Identifying bacteria in human urine: current practice and the potential for rapid, near-patient diagnosis by sensing volatile organic compounds. Clin Chem Lab Med 39(10):893-906. doi:10.1515/CCLM.2001.146

10. Dutta R, Hines EL, Gardner JW, Boilot P (2002) Bacteria classification using Cyranose 320 electronic nose. Biomed Eng Online 1:4. doi:10.1186/1475-925X-1-4

11. Lykos P, Patel PH, Morong CA, Joseph A (2001) Rapid detection of bacteria from blood culture by an electronic nose. J Microbiol 39(3):213-218

12. Aathithan S, Plant JC, Chaudry AN, French GL (2001) Diagnosis of bacteriuria by detection of volatile organic compounds in urine using an automated headspace analyzer with multiple conducting polymer sensors. J Clin Microbiol 39(7):2590-2593. doi:10.1128/ JCM.39.7.2590-2593.2001 